\title{
5R y Sustentabilidad hospitalaria: Nuestro aporte en la emergencia climática. Parte I. Reducir, Reutilizar y Reciclar
}

\author{
5R and Hospital Sustainability: Our contribution in the climate emergency. \\ Part I. Reduce, Reuse and Recycle
}

\author{
Waldo Merino MSc, MBA. ${ }^{1, *}$, Isabel Quispe PhD, MSc. ${ }^{2}$, Christian Guentelicán ${ }^{3}$, Sebastián Herrero ${ }^{3}$, Robin Estrada \\ Sebastián Inostroza ${ }^{3}$, Gonzalo Henríquez ${ }^{3}$, Claudio Cárcamo ${ }^{5}$, Francisco Rubilar MSc. ${ }^{5}$, Patricia Mogrovejo ${ }^{4}$, \\ 1 Departamento de Cirugía, Traumatología y Anestesia. Centro de Capacitación Investigación y Gestión en Salud para la Medicina \\ Basada en Evidencias (CIGES), Facultad de Medicina, Universidad de La Frontera. Temuco, Chile. \\ 2 Red Peruana de Ciclo de Vida y Ecología Industrial, Departamento de Ingeniería, Pontificia Universidad Católica del Perú. Lima, \\ Perú. \\ 3 Instituto del Medio Ambiente Universidad de La Frontera. Temuco, Chile. \\ 4 Interno de Medicina, Universidad de La Frontera. Temuco, Chile. \\ 5 Estudiante de Ingeniería Industrial, Pontificia Universidad Católica del Perú. Lima, Perú.
}

Financiamiento: Proyecto CF-HEALTHSUD (código 21- CLIMAT-03; Climat Amsud).

Agradecimientos: Se agradece a Fondecyt (Perú) por el soporte brindado.

Declaramos que ninguno de los autores de este trabajo presenta conflictos de interés.

Fecha de recepción: 22 de abril de 2021 / Fecha de aceptación 08 de julio de 2021

\begin{abstract}
Introduction: Climate change is a health problem and, at the same time, health systems are important contributors. Hospitals stand out due to their high rates of energy consumption, resources and waste generation. The purpose of the study is to know and identify the determinants of sanitary waste and the measures that can be implemented that allow reducing the production of hospital waste, seeking to achieve a general and updated appreciation of this phenomenon and taking into account hospital sustainability. Method: A bibliographic search was carried out in pubmed that included keywords related to the concepts of carbon footprint, recycling and hospital waste. The screening yielded a total of 37 articles and later 12 publications founded from references (or that were previously known by the authors) were added. Results: The results are presented into 5 points known as the " 5 Rs", named below. "Reduce" (through adequate segregation of waste, correct management of effluents and energy, significant reduction of excesses and automatic administration of anesthetic gases), "Reuse" (through device reprocessing, reusable material and donation), "Recycle", "Rethink" (with examples such as selection of less polluting gases, selective use of containers, staff education) and "Investigate" through different models. Discussion: Several of the recognized measures could have an application in many hospital areas despite the fact that most of the available evidence refers to the operating room. The organization and education of the personnel is important in order to implement the measures found.
\end{abstract}

Key words: Hospitals, medical waste disposal, recycling, equipment reuse, global warming.

\section{RESUMEN}

Introducción: La estrategia de disposición y generación de residuos influye en el cambio climático y éste, al mismo tiempo, afecta la salud de las personas, incrementando la frecuencia de enfermedades cardiovasculares, respiratorias e infecciosas, entre otras. Los sistemas sanitarios son contribuyentes importantes, debido a sus altas tasas de consumo de energía, recursos y generación de desechos. Esta revisión de la literatura pretende obtener una apreciación general y actualizada de los determinantes de desechos sanitarios y las medidas implementables para disminuir la producción de residuos hospitalarios y nuestro impacto en el medio ambiente. Método: Se realizó una amplia búsqueda bibliográfica en la base de datos Medline que incluyó palabras clave relacionadas a huella de carbono, reciclaje y residuos hospitalarios. El cribado arrojó un total de 48 publicaciones. Resultados: Se

waldo.merino@ufrontera.cl

https://orcid.org/0000-0002-4956-8444 
presentan los resultados organizados en 5 puntos, a los que se les conoce como las " 5 R" y que corresponden a "Reducir" (a través de segregación adecuada de residuos, correcta gestión de efluentes, energía y disminución de excesos), "Reusar" (a través de reprocesamiento de dispositivos), "Reciclar" (transformación de residuos), "Repensar" (búsqueda de nuevas formas, innovadoras y sustentables, de las prácticas clínicas) e "Investigar" (Research, generar nuevo conocimiento). Discusión: Numerosas estrategias pueden ser implementadas para contribuir a la sustentabilidad hospitalaria y podrían tener una aplicación en muchas áreas, a pesar de que la mayoría de la evidencia disponible hace referencia al sector quirúrgico. La investigación nos ofrece herramientas para desarrollar nuevas alternativas de gestión, donde la organización y educación del personal son esenciales.

Palabras clave: Hospitales, eliminación de residuos sanitarios, reciclaje, equipo reutilizado, calentamiento global.

\section{Introducción}

a Organización Mundial de la Salud (OMS) en el año 2015 hace referencia al cambio climático, como el problema más importante para los sistemas de salud en el siglo XXI[1]. Los riesgos proyectados para la salud pública global, debido a eventos extremos como olas de calor, sequías, inundaciones, incendios y amenazas a la producción de alimentos están relacionados al cambio climático[2], incrementando la mortalidad directa o indirecta por desnutrición, enfermedades cardiorrespiratorias, enfermedad diarreica y cambios en la distribución de patógenos infecciosos[3]. De manera paradójica, los sistemas sanitarios son contribuyentes importantes en términos de contaminación, por ejemplo, el sistema de salud de Estados Unidos durante 2013 fue responsable del 9,8\% de los gases de efecto invernadero[4].

Una arista en que los hospitales contribuyen de manera considerable al impacto ambiental, es a través de altas tasas de consumo de energía, recursos y generación de residuos, debido a que frecuentemente utilizan gran cantidad de productos desechables[5].

En Australia, el sistema de salud es el segundo mayor contribuyente de desechos, luego de la industria alimentaria, con 236 mil toneladas de desechos por año. La situación se repite en el Reino Unido y Estados Unidos, donde existen instalaciones que producen entre 181 mil y 1,8 millones de toneladas de residuos por año[5]

Dentro de un hospital, el área quirúrgica se destaca por una actividad con alta necesidad y utilización de equipamiento, procesos de esterilización, tecnologías operativas avanzadas y sistemas de soporte vital obligatorio. Estas actividades, utilizan gran cantidad de energía y generan grandes volúmenes de residuos[6]. En la actualidad, existe un incremento en el interés de "ecologizar" el ambiente perioperatorio para reducir los costos ambientales, con objeto de mitigar su impacto, sin afectar la calidad de la atención médica.

El propósito de este artículo es, por medio de una búsqueda bibliográfica amplia, conocer e identificar los determinantes de desechos sanitarios y las medidas implementables que permitan disminuir la generación de residuos de manera de alcanzar una apreciación general y actualizada de este fenómeno, teniendo en cuenta la sustentabilidad hospitalaria.

\section{Material y Método}

Se realizó una búsqueda que incluyó palabras claves relacionadas al cambio climático, como son huella de carbono, reciclaje y residuos hospitalarios. Para ello se realizó la siguiente búsqueda en MEDLINE: ("Hospitals"[Mesh] OR "Operating Rooms"[Mesh] OR "Surgical Procedures, Operative"[Mesh] OR "Anesthesia"[Mesh]) AND ("Medical Waste Disposal"[Mesh] OR "Recycling"[Mesh] OR "Waste Management"[Mesh] OR "Product Packaging"[Mesh]) AND ("Environmental Monitoring"[Mesh] OR "Global Warming"[Mesh] OR "Equipment Reuse"[Mesh] OR "Recycling"[Mesh] OR "Waste Management"[Mesh]) se aplicaron los filtros: 5 años, inglés, español, humanos. 145 resultados. Posteriormente, se realizó un cribado de los artículos en base al título y abstract disponible con los siguientes criterios de inclusión: Artículos que mencionan medidas tomadas para reducir, reutilizar o reciclar en servicios hospitalarios. Artículos que mencionan el impacto medioambiental de estas medidas o la disminución de residuos generadas. El cribado arrojó 37 documentos en total, de los cuales se realizó una lectura completa, se seleccionaron aquellos que aportaban información relevante, actualizada y/o novedosa. Posteriormente, se agregaron un total de 12 publicaciones más, a partir de las referencias de los artículos obtenidos o que eran de conocimiento de los autores.

\section{Resultados}

\section{Residuos y Metodología 5Rs}

La ecología propone estrategias para la minimización de residuos conocidas como las $3 \mathrm{R}$, las cuales son: Reducir, Reutilizar y Reciclar. Actualmente, se habla de $5 \mathrm{R}$, debido a la incorporación de Repensar e Investigar (del inglés Research). Estos principios, propuestos sobre hábitos de consumo, pueden ser equiparables y utilizados en los servicios clínico-hospitalarios [7]. En esta primera parte se presentarán las primeras y clásicas 3 R.

A continuación, utilizando la propuesta por Kagoma y cols., en "People, planet and profits: the case for greening operating rooms"[7], se describe los resultados de la búsqueda en cada una de estas 5 categorías temáticas (Figura 1).

Para trabajar los aspectos relacionados a las $5 R$ es necesario conceptualizar qué es un residuo. Un residuo o desecho es toda sustancia que el generador (persona o actividad que origina residuos) elimina, se propone eliminar o está obligado a eliminar. Los residuos generados en establecimientos de atención en salud se clasifican en 4 categorías: Residuos peligrosos, radiactivos de baja intensidad, especiales y sólidos asimilables a domiciliarios. Si bien la clasificación depende de las entidades, esto está determinado por el marco legal en cada país. El marco 


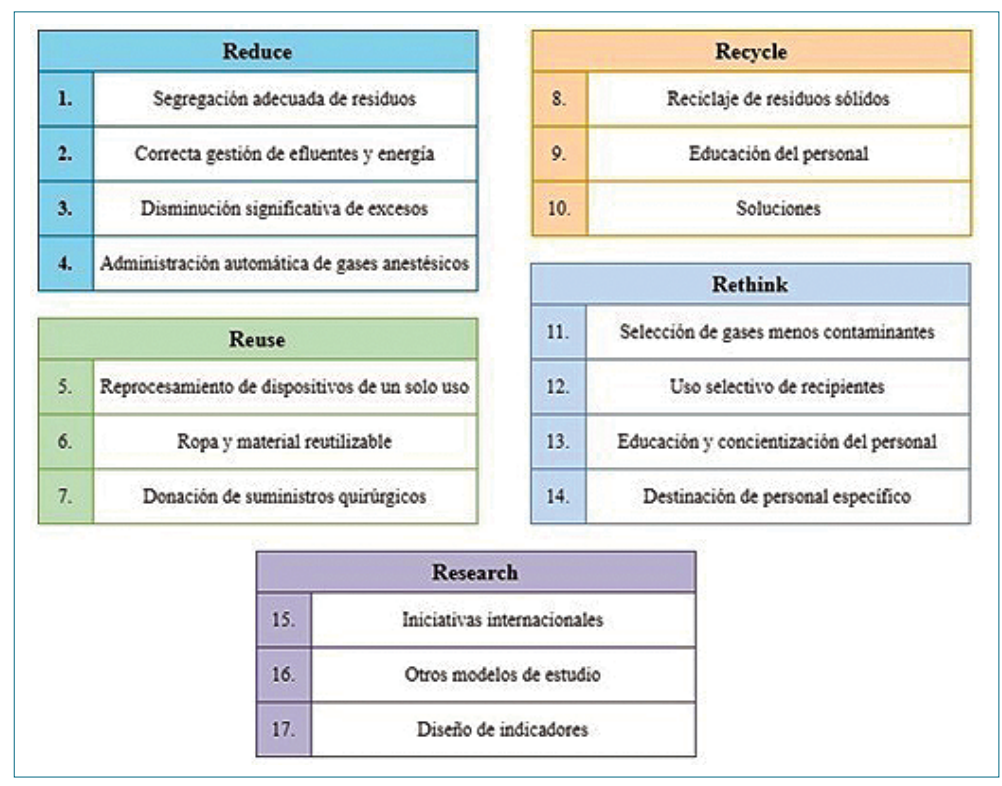

Figura 1. Categorías temáticas de cada "R".

legal es importante principalmente para el manejo de los residuos peligrosos, especiales y radioactivos y en este suelen intervenir ministerios $u$ organizaciones especializadas para verificar que se cumpla la normativa y evitar peligros[8].

Residuo peligroso, residuo o mezcla de éstos que presenta riesgo para la salud pública y/o efectos adversos al medio ambiente, ya sea directamente o debido a su manejo, como consecuencia de presentar algunas de las siguientes características: toxicidad aguda, crónica e intrínseca, inflamabilidad, reactividad o corrosividad. Los medicamentos, drogas y productos farmacéuticos son considerados peligrosos, a menos que su generador pueda demostrar ante la autoridad sanitaria que no presenta característica de peligrosidad.

Residuos especiales, aquellos sospechosos de contener agentes patógenos en concentración suficiente para causar enfermedad e incluyen cultivos, muestras almacenadas, restos biológicos, sangre y productos derivados, cortopunzantes y residuos animales.

Residuos sólidos asimilables a domicilio, aquellos que por sus características pueden ser entregados a la recolección municipal y dispuestos en relleno sanitario. Dentro de esta categoría se encuentran: Preparación y servicio de alimentos, material de limpieza, papel, materiales de oficina y materiales absorbentes.

El manejo de los residuos asociados a atención en salud (REAS) incluye su almacenamiento, transporte y eliminación. El manejo interno, realizado al interior del establecimiento de salud, comprende: 1) Segregación adecuada en contenedores identificados y etiquetados para cada categoría; 2) Recolección y transporte desde la fuente de generación hasta la sala de almacenamiento; 3) Almacenamiento, de acuerdo a las categorías de segregación.

El manejo externo contempla: 1) Recolección y transporte, mediante el retiro de los residuos, en forma separada, a instalaciones de recuperación, tratamiento o disposición final que cuenten con la autorización sanitaria; 2) Disposición final y eliminación que comprende las operaciones mediante las cuales los residuos son tratados y dispuestos en el suelo definitivamen- te, esta etapa se realiza generalmente fuera del establecimiento y operada por terceros, sin embargo, algunos hospitales por su complejidad y magnitud pueden contar en sus instalaciones con sistemas de tratamiento de residuos. Cabe destacar que la eliminación de residuos peligrosos debe cumplir con el Decreto Supremo N¹48/2003 del Ministerio de Salud y todo establecimiento que los elimine debe contar con permiso de la Autoridad Sanitaria(8).

\section{Aplicación de la Metodología 5 Rs en hospitales}

\subsection{Reducir}

Hace referencia a minimizar la cantidad de residuos que pasarán a una disposición final como son los rellenos sanitarios o rellenos de seguridad. Para lograr reducir, se utilizan las siguientes acciones.

Segregación adecuada de residuos. Las políticas de reducción de residuos han sido aplicadas, principalmente, por medio de dos medidas: 1) Programas de educación básica y capacitación, acerca del impacto ambiental de los residuos y su correcta clasificación; 2) Modificación en la cantidad de contenedores, aumentando los de residuos generales y disminuyendo los de residuos especiales.

Diversos estudios, muestran que a través de estas políticas han demostrado una reducción variable en la generación de residuos y un consecuente ahorro de recursos en la disposición final. Solo aplicando un programa de educación es posible reducir la generación de residuos desde 6,2\% hasta $67 \%$ dependiendo de las medidas implementadas[9], cifra que puede alcanzar $82 \%$ cuando se asocia a un programa de reciclaje[5].

Se identificó como factores asociados a buenas prácticas (Reducir) el tener menos de 2 años de experiencia laboral, estar informado en precauciones estándar y la presencia de contenedores separados para los diferentes residuos[10].

Los países con un PIB más alto poseen una mayor tasa de generación de residuos, sin embargo, se asocian a mejores políticas ambientales y menor cantidad de residuos peligrosos sobre los no peligrosos, contrario a lo que ocurre en países en 
vías de desarrollo, donde esta relación (residuos peligrosos/no peligrosos) aumenta[10].

Con respecto al manejo de los REAS, existe un manual desarrollado por el gobierno en el que se detalla un color para la segregación de cada categoría de residuo como se muestra en la (Figura 2)[11].

Menor consumo de agua. Jehle y cols.[12] exploraron formas de reducir el desperdicio de agua en el entorno perioperatorio y descubrieron que, en promedio, 3,25 miembros del personal se lavan para un caso quirúrgico y se utilizan más de 60 litros de agua para el lavado quirúrgico de manos. Controversialmente, abogaron por una técnica de exfoliación al comienzo con productos alcohólicos, siempre que las manos no estén visiblemente sucias, (recomendación del Instituto Nacional de Salud y Excelencia en la Atención del Reino Unido) sumado a la instalación de un sistema intermitente de agua corriente, este último aspecto es un punto crítico en el consumo de agua de todo el recinto hospitalario[12].

Menor gasto de energía. Implementación de iniciativas de reducción de consumo de energía tales como, apagar luces y equipos hospitalarios que no estén en uso, lo cual ahorró aproximadamente 33.004 dólares al año y redujo 243 toneladas métricas de $\mathrm{CO}_{2 \text { eq }}$ en un hospital en Carolina del Norte[13].

Entre las sugerencias para reducir el consumo de electricidad se encuentra el uso dispositivo de iluminación de bajo consumo, sensores de ocupación para áreas públicas, controles de calefacción hidrónicos y películas solares en las ventanas[14].

Reducción del exceso. Muchos artículos, desde el primer mundo, también enfatizan el beneficio de donar materiales no utilizados como ayuda a los países en desarrollo como un medio para desviar los desechos del vertedero y reducir los costos de eliminación, pero se destaca que este tipo de iniciativa po- dría, en algunos casos, significar finalmente el traslado desde un vertedero a otro si el receptor no posee una política para el manejo de estos equipos[5].

Otra forma de reducir el exceso, es la disminución de la cantidad de instrumentos dispuestos en las bandejas quirúrgicas, haciéndolas más personalizadas para el procedimiento a realizar. Esta medida se aplicó a 1.099 procedimientos, ahorrando 13.250 dólares y una reducción de 2,8 toneladas de residuos[15] o una reducción de 0,3 kilogramos de residuos por cirugía (13\% menos de residuos generados)[17].

Se ha recomendado, para reducir activamente el desperdicio y los costos de suministros quirúrgicos, que el personal perioperatorio conozca el precio de cada suministro. Una forma de lograr este objetivo es agregar el costo de cada producto en una etiqueta, a vista del personal médico, que le permita relacionar el costo del artículo con su uso[16].

Uso de control de administración de gases anestésicos automático. Está bien descrita la mayor eficiencia del uso de control de gas automático en población adulta con ejemplos como la máquina Zeus ${ }^{\circledR}$ donde el modo automático provee una similar estabilidad hemodinámica y control del índice biespectral (BIS) a la vez que disminuye a más de la mitad el consumo de oxígeno y desfluorano[17]" o la "Maquet Flowi anesthesia machine (Maquet, Solna, Sweden)" en adultos cuyo control de gas automático otorga un patrón decreciente de flujo de gas fresco y permite además seleccionar una $\mathrm{FiO}_{2}$ objetiva[18].

En población pediátrica un estudio utilizó la "Maquet Flow-i anesthesia machine (Maquet, Solna, Sweden)" y comparó por 4 semanas el gasto de sevofluorano de este equipo con la administración de forma tradicional, reduciendo un tercio su uso (en 220 casos y 230 horas de anestesia)[19].

\begin{tabular}{lll|}
\hline Categoría de residuo & Color & Símbolo \\
Residuos especiales & Amarillo & \\
Residuos peligrosos & $\begin{array}{l}\text { Cualquiera, excepto } \\
\text { amarillo,negro o gris }\end{array}$ & $\begin{array}{l}\text { De acuerdo a la característica de } \\
\text { Oeligrial. } \\
\text { Residuos radiactivos de Norma Chilena }\end{array}$ \\
baja intensidad & $\begin{array}{l}\text { Cualquiera, excepto } \\
\text { amarillo, negro o gris }\end{array}$ \\
$\begin{array}{l}\text { Residuos sólidos } \\
\text { asimilables }\end{array}$ & Gris o negro \\
& &
\end{tabular}

Figura 2. Segregación por color y símbolo según categoría de residuos. 


\subsection{Reutilizar}

Reutilizar hace referencia a sustituir materiales desechables por materiales reutilizables. Algunos ejemplos incluyen ropa quirúrgica, almohadillas y tubos de succión.

Reutilización de dispositivos de un solo uso. Una opción para reducir el impacto ambiental de los artículos desechables es la reutilización de dispositivos de un solo uso (jeringa de irrigación, aguja de jeringa, hojas de bisturí, trócar, grapadora, esponjas). Éstos se recolectan en contenedores exclusivos, se limpian, prueban, reesterilizan y empaquetan[20]. A través de esta práctica algunos autores han reportado reducciones superiores a las 5 toneladas de desechos y más de 4.000 dólares de ahorro anuales en costos con variados materiales[13]. Otra consideración encontrada es que al comparar materiales no reutilizables v/s reutilizables en pabellón, estos últimos resultaron superiores en facilidad de uso, confort y propiedades de protección según profesionales encuesta$\operatorname{dos}[21]$

Ropa y material reutilizable. Las batas estériles reutilizables han significado una reducción de $23.000 \mathrm{~kg}$ de residuos anuales y ahorro de 60.000 dólares[22]. De igual forma, la utilización de almohadillas de gel reutilizable ha mostrado ahorrar más de 50.000 dólares anuales[13].

Por otro lado, algunos estudios han mostrado resultados negativos relativos a la huella de carbono y el uso de material reutilizable, por ejemplo McGain y cols.[23], muestra que, en un hospital en Australia, reemplazar el material de uso único por reutilizable aumentó casi en 10\% las emisiones de $\mathrm{CO}_{2 \text { eq }}$ en el análisis del ciclo de vida (LCA) de los productos, sin embargo, los costos disminuyeron 23.000 dólares al año[23]. En términos de reutilización de materiales, el escenario difiere ampliamente entre cada país, así como sus leyes en referencia al reuso de los dispositivos de uso único. Si un producto puede ser reutilizado con protocolos validados y manteniendo su funcionalidad no hay razones para darle un solo uso[24].

Donación. Tal como la donación de suministros es una acción a considerar para la reducción del exceso, también es aplicable al reutilizar, debido a que, parte de los desechos provienen de equipos estériles pre empaquetados y parcialmente usados. La mayoría de las misiones recolectan suministros donados, tales como apósitos cerca de la fecha de vencimiento, gasas, kits intravenosos, antimicrobianos y tubing supplies[25]. La Organización Mundial de la Salud recomienda cuatro principios para una buena donación: Respetar la solicitud del receptor, asegurarse que la donación será beneficiosa, mantener una línea de comunicación abierta y garantizar la calidad[26].

\subsection{Reciclar}

Potencial de reciclaje. Existe potencial de reciclaje en distintas áreas de los centros de salud, destacando el pabellón y la cirugía ortopédica.

Debido a que es una práctica común, en muchos hospitales en el Reino Unido, clasificar todos los desechos del pabellón quirúrgico como desechos clínicos, Southorn y cols., decidieron medir el peso de los desechos para operaciones ortopédicas (reemplazo total de cadera, reemplazo total de rodilla e inyección de articulación facetaria), evidenciando que cerca del 50\% correspondía a desechos domésticos, de los cuales $47 \%$ era reciclable, $47 \%$ potencialmente reciclable y solo $6 \%$ definitiva- mente no reciclable[27]. En Canadá se describió que, dentro de la ortopedia, la artroplastia es una de las cirugías con la mayor tasa de generación de residuos potencialmente reciclables y por tanto postulaban énfasis en el reciclaje efectivo durante esta operación[28].

McGain y cols., realizaron una auditoría prospectiva de 1 semana de todos los desechos de 6 pabellones en el Western Hospital, Melbourne durante el 2012. Se instalaron contenedores para papel/cartón, plásticos mixtos y polipropileno. Se observó que en 237 operaciones se generaron 1.265 kg de residuos, dentro de éstos los desechos reciclables fueron $23 \%$. La tasa de reciclaje global alcanzada en relación con el potencial fue de 285/517 kg, correspondiente a 55\%. Más allá de reducir la cantidad de desechos infecciosos, los beneficios financieros no fueron significativos[29].

Educación del personal. A su vez existe desconocimiento relacionado al reciclaje, en una encuesta realizada en la "Mayo Clinic" 56,7\% afirmó no tener claro qué artículos del pabeIlón son reciclables y el 47,7\% señaló como principal barrera para el reciclaje la falta de conocimientos[30]. Un estudio que encuestó a 780 anestesiólogos de Nueva Zelanda, Australia e Inglaterra con respecto al reciclaje en pabellón mostró que sólo $11 \%$ de los anestesiólogos consideraban que se produjo un reciclaje adecuado. Las principales dificultades que encontraron fueron instalaciones de reciclaje inadecuadas, actitudes negativas por parte del personal y falta de información sobre cómo reciclar[31]. Al implementar un programa educativo simple de reciclaje, en un centro de cirugía estética con 3 pabellones en Arizona, se disminuyeron en $12 \mathrm{~kg}$ por mes los residuos cortopunzantes, lo que representó un ahorro de costos del $10,3 \%[30]$.

Experiencias de reciclaje. En un departamento de neurocirugía implementaron un proyecto de 8 semanas donde vendieron a empresas de reciclaje las envolturas azules ("blue wraps") usadas en pabellón. En las 8 semanas se recolectaron $565 \mathrm{~kg}$ en total, con lo que consiguieron más de 30.000 dólares en ahorro y estimaron que al año se ahorrarían más de 170.000 dólares[32].

En Escocia McKendrick y cols., describieron un método pragmático para reciclar papel y cartón en la sala de preparación para el pabellón y la sala de anestesia. Los resultados mostraron que la sala de anestesia producía un peso de 1,3 kg por paciente (50\% papel y cartón) y la sala de preparación producía 3,5 kg por paciente (33\% residuo general, 44\% papel, $23 \%$ cartón). El método de reciclaje ahorró en promedio 1,37 dólares por caso y las $54 \mathrm{~kg}$ de bolsas de reciclaje producidas durante el estudio disminuyeron $25 \mathrm{~kg}$ de emisiones de $\mathrm{CO}_{2 \text {-eq }}$ [33].

En Suecia todo metal recolectado en cremación se recicla desde 2016, por lo que se ha sugerido la iniciativa de reciclar los implantes óseos o articulares bajo auspicio de sociedades ortopédicas, con el fin de asumir su responsabilidad medioambiental y generar recursos económicos que podrían utilizarse en investigación[34].

Todo lo mencionado hasta el momento puede (y debería) ser integrado en los proyectos de sustentabilidad. Un ejemplo está en el Gundersen Health System (Wisconsin) que en 2015 recicló, reutilizó y desvió el $46 \%$ de su flujo de desechos sólidos, resultando en un reembolso de más de 70.000 dólares. Dentro de sus medidas está el reciclaje de 480 toneladas de pa- 
pel, cartón y plástico, donación de 78 toneladas de suministros médicos a organizaciones de caridad y donación de alimentos al ejército de salvación local[35].

\section{Referencias}

1. Sherman J, Le C, Lamers V, Eckelman M. Life cycle greenhouse gas emissions of anesthetic drugs. Anesth Analg 2012 May;114(5):1086-90. https://doi.org/10.1213/ ANE.0b013e31824f6940 PMID:22492186

2. Ramanathan $V$, Haines $A$. Healthcare professionals must lead on climate change. BMJ. 2016 Oct;355:i5245. https://doi. org/10.1136/bmj.i5245 PMID:27702748

3. Costello A, Abbas M, Allen A, Ball S, Bell S, Bellamy R, et al. Managing the health effects of climate change. Lancet. 2009 May;373(9676):1693-733. https://doi.org/10.1016/S01406736(09)60935-1 PMID:19447250

4. Eckelman MJ, Sherman J. Environmental Impacts of the U.S. Health Care System and Effects on Public Health. PLoS One. 2016 Jun;11(6):e0157014. https://doi.org/10.1371/journal. pone.0157014 PMID:27280706

5. Wyssusek KH, Keys MT, van Zundert AA. Operating room greening initiatives - the old, the new, and the way forward: A narrative review. Waste Manag Res. 2019 Jan;37(1):3-19. https://doi. org/10.1177/0734242X18793937 PMID:30132405

6. Macneill AJ, Lillywhite R, Brown CJ. Articles The impact of surgery on global climate : a carbon footprinting study of operating theatres in three health systems. Lancet Planet Heal. 2017;1(9):e3818. Disponible en: https://doi.org/10.1016/\$2542-5196(17)301626.

7. Kagoma YK, Stall N, Rubinstein E, Naudie D. People, planet and profits: the case for greening operating rooms. CMAJ. 2012 Nov; 184(17):1905-11. https://doi.org/10.1503/cmaj.112139 PMID:22664760

8. Ministerio de Salud. Reglamento sobre Manejo de Residuos de Establecimientos de Atención en Salud (REAS). D Of. 2009; Disponible en: https://www.minsal.cl/sites/default/files/files/REAS.pdf

9. Mosquera M, Andrés-Prado M, Rodríguez-Caravaca G, Latasa P, Mosquera M. American Journal of Infection Control Evaluation of an education and training intervention to reduce health care waste in a tertiary hospital in Spain. Am J Infect Control. 2014;1-4. Disponible en: https://doi.org/10.1016/j.ajic.2014.04.013.

10. Ansari M, Hassan M, Farzadkia M, Ahmadi E. Dynamic assessment of economic and environmental performance index and generation, composition, environmental and human health risks of hospital solid waste in developing countries; A state of the art of review. Environ Int. 2019; 132:105073. Disponible en: https:// doi.org/10.1016/j.envint.2019.105073.

11. Ministerio de Salud. Manejo de residuos de establecimientos de atención de salud. Santiago, Chile; 2010. pp. 1-88.

12. Jehle K, Jarrett N, Matthews S, Surgery G, Theatres O, Hospital W. Clean and green: saving water in the operating theatre. Ann R Coll Surg Engl. 2008 Jan;90(1):22-4. https://doi. org/10.1308/003588408X242277 PMID:18201493

13. Wormer BA, Augenstein VA, Carpenter CL, Burton PV, Yokeley WT, Prabhu AS, et al. The green operating room: simple changes to reduce cost and our carbon footprint. Am Surg. 2013 Jul;79(7):666-71. https://doi.org/10.1177/000313481307900708 PMID:23815997.
14. Kaplan S, Sadler B, Kevin L, Franz C, Orris P. Can Sustainable Hospitals Help Bend the Health Care Cost Curve? Commonw Fund; 2012. p. 29.

15. Demark RE Van, Smith V, Fiegen A. Lean and Green Hand Surgery. J Hand Surg Am. 2018;43(2):179-81. Disponible en: https:// doi.org/10.1016/j.jhsa.2017.11.007.

16. Putman K. Surgical supply waste: putting it all on the table. Assoc Perioper Regist Nurses J. 2016;103(4):10-1.

17. Lortat-Jacob B, Billard V, Buschke W, Servin F. Assessing the clinical or pharmaco-economical benefit of target controlled desflurane delivery in surgical patients using the Zeus anaesthesia machine. Anaesthesia. 2009 Nov;64(11):1229-35. https://doi. org/10.1111/j.1365-2044.2009.06081.x PMID:19825059

18. Carette R, De Wolf AM, Hendrickx JF. Automated gas control with the Maquet FLOW-i. J Clin Monit Comput. 2016 Jun;30(3):341-6. https://doi.org/10.1007/s10877-015-9723-6 PMID:26072157

19. Moran P, Barr D, Holmes C. Saving Sevoflurane: Automated gas control can reduce consumption of anesthetic vapor by one third in pediatric anesthesia. Pediatr Anesth. 2019;29(4):0-2.

20. Sherman JD, Ryan S. Ecological responsibility in anesthesia practice. Int Anesthesiol Clin. 2010;48(3):139-51. https://doi. org/10.1097/AIA.0b013e3181ea7587 PMID:20616643.

21. Conrardy J, Hillanbrand M, Myers S, Nussbaum GF. Reducing Medical Waste. Assoc Perioper Regist Nurses J. 2010;91(6):711-21. Disponible en: https://doi.org/10.1016/j.aorn.2009.12.029.

22. Kwakye G, Brat GA, Makary MA. Green surgical practices for health care. Arch Surg. 2011 Feb;146(2):131-6. https://doi. org/10.1001/archsurg.2010.343 PMID:21339421.

23. McGain F, Story D, Lim T, McAlister S. Financial and environmental costs of reusable and single-use anaesthetic equipment. $\mathrm{Br}$ Anaesth. 2017 Jun;118(6):862-9. https://doi.org/10.1093/bja/ aex098 PMID:28505289.

24. Hussain M, Balsara KP, Nagral S. Reuse of single-use devices: looking back, looking forward. Natl Med J India. 2012 MayJun;25(3):151-5. PMID:22963293.

25. Guetter CR, Williams BJ, Slama E, Arrington A, Henry MC, Möller MG, et al. Greening the operating room. Am J Surg. 2018;216(4):683-8. Disponible en: https://doi.org/10.1016/j. amjsurg.2018.07.021.

26. World Health Organization. Guidelines For Health Care Equipment Donations. World Heath Organ. 2000;(March):1-30.

27. Southorn T, Norrish AR, Gardner K, Baxandall R. Reducing the carbon footprint of the operating theatre: a multicentre quality improvement report. J Perioper Pract. 2013 Jun;23(6):1446. https://doi.org/10.1177/175045891302300605 PMID:23909168

28. Kooner S, Hewison C, Sridharan S, Lui J, Matthewson G, Johal $\mathrm{H}$, et al. Waste and recycling among orthopedic subspecialties. Can J Surg. 2020;63(3): E278-83. Disponible en: https://doi. org/10.1503/cjs.018018.

29. McGain F, Jarosz KM, Nguyen MNHH, Bates S, O'Shea CJ. Auditing Operating Room Recycling: A Management Case Report. A case reports. 2015;5(3):47-50.

30. Azouz S, Boyll P, Swanson M, Castel N, Maffi T, Rebecca AM. Managing barriers to recycling in the operating room. Am J Surg. 2019;217(4):634-8. Disponible en: https://doi.org/10.1016/j. amjsurg.2018.06.020.

31. McGain F, White S, Mossenson S, Kayak E, Story D. A survey of anesthesiologists' views of operating room recycling. Anesth 
Analg. 2012 May;114(5):1049-54. https://doi.org/10.1213/ ANE.0b013e31824d273d PMID:22415530

32. Babu MA, Dalenberg AK, Goodsell G, Holloway AB, Belau MM, Link MJ. Greening the Operating Room: Results of a Scalable Initiative to Reduce Waste and Recover Supply Costs. Neurosurgery. 2019 Sep;85(3):432-7. https://doi.org/10.1093/neuros/nyy275 PMID:30060055

33. McKendrick DR, Snedden LJ, Bunch R, McGregor H. Pragmatic recycling of paper and cardboard in the operating theatre: an audit. J Perioper Pract. 2017 Mar;27(3):43-8. https://doi. org/10.1177/175045891702700302 PMID:29328742

34. Lidgren L, Raina DB, Tägil M, Tanner KE. Recycling implants: a sustainable solution for musculoskeletal research. Acta Orthop. 2020 Apr;91(2):125. https://doi.org/10.1080/17453674.2019.17 06301 PMID:31902268

35. Ferenc J. Gundersen's recycling pays off in more ways than one. 2016. Disponible en: https://www.hfmmagazine.com/ articles/2247-gundersen-health-recycling-program-pays-off 